\title{
Competencia manejo del tiempo en la formación de profesionales de la administración
}

\author{
Durán-Aponte, Emilse*
}

\begin{abstract}
Resumen
En la actualidad adquiere más fuerza la necesidad de incorporar a la formación universitaria de los profesionales de la administración las competencias para afrontar la dinámica organizacional y social en la que se van a involucrar. En función de ello se efectuó un estudio correlacional, con el objetivo de describir las dimensiones de la competencia genérica manejo del tiempo en estudiantes universitarios de carreras administrativas; todos ellos inscritos en los últimos cursos de su plan de estudios. Participaron 278 estudiantes de los cuales 173 (62,2\%) eran mujeres y 105 (37,8\%) hombres. Se aplicó el cuestionario de manejo del tiempo (TMBQ-1990). El 84,76\% afirma que no recibió información sobre manejo del tiempo y presentan una correlación $(r=, 129, p=, 005)$ con la dimensión preferencia por la desorganización. Se encontraron diferencias estadísticamente significativas entre mujeres y hombres solo en la dimensión herramientas de gestión (Diferencia de medias=-,195, $p<001$ ), en donde las mujeres presentan mayores puntajes. Se concluye que los estudiantes presentan puntajes aceptables en cuanto a su manejo del tiempo; sin embargo, es necesario incrementar los esfuerzos por la formación en esta y otras competencias genéricas en los futuros profesionales.
\end{abstract}

Palabras clave: Manejo del tiempo, competencias genéricas, formación universitaria, profesiones administrativas.

\section{The Time Management Competence in Training Management Professionals}

\begin{abstract}
Today, the need university education for management professionals has to incorporate competences that prepare students to face the social and organizational dynamics in which they will be involved is gathering force. Based on this, a correlational study was conducted with the aim of describ-

Recibido: 06-09-11. Aceptado: 02-04-12

* $\quad$ Lic. Educación. Magíster en Psicología. Estudiante del Doctorado en Ciencias Sociales y Humanidades. Universidad Simón Bolívar (USB). Profesora del Departamento de Formación General y Ciencias Básicas, Universidad Simón Bolívar. e-mail: emilseaponte@usb.ve.
\end{abstract}


ing dimensions of the generic competence "time management" in university administration students enrolled in their final years of study. Of the 278 students participating, $173(62.2 \%)$ were female and 105 (37.8\%) male. The Time Management Behavior Questionnaire (TMBQ-1990) was applied. $84.76 \%$ said they received no information about time management and are correlated $(r=129, p=$, $005)$ with the preference for disorganization dimension. Statistically significant differences were found between women and men, only in the management tools dimension (Mean Difference $=-, 195$, $p<001$ ), where women had higher scores. Conclusions are that students have acceptable scores in terms of time management; nevertheless, training efforts should be increased for this and other generic competences for future professionals.

Keywords: Time management, generic competences, college education, administrative professions.

\section{Introducción}

La formación universitaria busca entre otras cosas, capacitar a los individuos para el desempeño profesional, aunque según afirma Rodríguez et al., (2010), esta transición no se da de manera inmediata, en algunos casos debido a la crisis global, las altas tasas de desempleo, la competitividad, la automatización de los puestos de trabajo, y otros factores; en general se espera que los egresados universitarios puedan conseguir un puesto de trabajo y desempeñarse bien laboralmente.

Sumado a la poca oferta de empleos, el contexto laboral en el que nos desenvolvemos en la actualidad está caracterizado por lo variante, urgente, rápido y a veces impredecible de los eventos que suceden (Castellano, 2010).

Esta realidad demanda en los que egresan de las aulas universitarias, una formación que responda ante los retos actuales y los prepare para afrontar el cambio y la incertidumbre, a la vez que se hagan competentes ante las características de la dinámica organizacional y social en la que se van a involucrar y que cuenten con las herramientas para hacerlo.
Una formación universitaria en profesiones del área administrativa e incluso cualquier área, consciente de este contexto deberá tener en cuenta que se promueva el desarrollo de competencias (genéricas y profesionales) en los futuros egresados, que les permitan ser competitivos en el ambiente laboral, sin dejar de lado su crecimiento personal y el aporte que pueden brindar con su trabajo a la sociedad.

Una de las competencias genéricas más solicitada por los empleadores para acceder a los puestos de trabajo, según investigaciones recientes de Martínez (2011), Tejeda (2011), Rodríguez et al. (2010), y Olvera (2008), es el manejo eficaz y eficiente del tiempo, según estos autores esta competencia se relaciona con la capacidad para adaptarse a ambientes cambiantes con una actitud positiva, tolerancia al estrés, manejo de la incertidumbre y trabajo bajo presión, características que definen los actuales puestos de trabajo a nivel de Iberoamérica y el resto del mundo.

Esta competencia adquiere mayor relevancia cuando se trata de la formación de futuros profesionales en áreas relacionadas con las ciencias administrati- 
vas, para desempeñarse en ámbitos específicos como la administración de empresas aduanales nacionales e internacionales, empresas de transporte, comercio exterior, administración de empresas turísticas y hoteleras y organización empresarial, carreras dictadas en la Universidad Simón Bolívar institución en la que se encuentra la muestra en estudio.

El perfil profesional de estos futuros egresados demanda una conducta eficaz para planificar, resolver problemas, tomar decisiones, manejar el estrés, gestionar el talento humano, gestionar recursos materiales y financieros y otros elementos afines, en medio de un ambiente dinámico, competitivo y de presión. Si a esto se suma que el futuro egresado también debe ocuparse de su desarrollo personal, participar en actividades fuera del trabajo, integrar y participar en la dinámica familiar y responder al contexto social, nos estamos refiriendo a un profesional capaz de responder a su entorno personal, laboral y social de manera equilibrada y responsable, sin desestimar ninguna de estas áreas.

Debido a lo expuesto anteriormente, los individuos que cursan estas carreras además de contar con competencias técnicas o específicas asociadas a la profesión, requieren también la competencia de gestionar el tiempo para desempeñarse de manera exitosa, por cuanto se considera un elemento clave para destacar en su vida personal y por consiguiente en ambiente laboral (Whetten y Cameron, 2005). Bajo este enfoque, esta investigación tiene por objetivo describir las dimensiones de la competencia manejo del tiempo en estudiantes universitarios de carreras administrativas; todos ellos inscritos en los últimos cursos de su plan de estudios.

Tal como lo indican Tejeda y Sánchez (2009: 43), "en una formación basada en competencias profesionales en el nivel universitario es imprescindible diagnosticar y trabajar a partir de las potencialidades del discente...", por lo tanto se justifica esta investigación considerando la importancia de identificar cuáles son los patrones de manejo del tiempo en los futuros profesionales, lo que permitirá profundizar en el estudio de esta competencia de forma científica para determinar si es necesario, realizar futuras intervenciones y asesorías orientadas a promoverla en la formación universitaria, e incluso proponer incorporaciones a nivel de planes de estudios o asignaturas de formación general orientadas al desarrollo de competencias genéricas.

Los resultados que se generen ayudarán a los empleadores a comprender mejor los perfiles de quienes aspiran a los puestos de trabajo y además, se convertirán en una opción de consulta para otros investigadores interesados en la formación por competencias, y en específico en la medición de la competencia genérica manejo del tiempo.

Así mismo, conocer cómo se da el manejo del tiempo en los futuros egresados, permitirá analizar si la intervención o proceso de actuación llevado a cabo en la institución donde cursan estudios universitarios, no solamente es correcto sino también eficaz, tal como lo sugiere Cepeda (1994).

La metodología empleada fue de tipo descriptivo-correlacional, realizada 
Competencia manejo del tiempo en la formación de profesionales de la administración Durán-Aponte, Emilse

con un diseño no experimental (Kerlinger y Lee, 2008) y transeccional, puesto que la información se recolectó a través de cuestionarios aplicados en un solo momento de administración a la muestra (Hernández et al., 2003).

Participaron 278 estudiantes cursantes de los dos últimos períodos académicos antes de culminar los estudios de carrera en la Universidad Simón Bolívar, de los cuales 173 (62,2\%) eran mujeres y $105(37,8 \%)$ hombres. Sus edades están entre 18 y 25 años, con una media de 20 años y una desviación típica de 1,54. Las carreras en orden de magnitud fueron Administración de Aduanas, Comercio Exterior, Organización Empresarial, Administración del Turismo, Administración del Transporte y Administración Hotelera.

Las dimensiones de manejo del tiempo se midieron a través del cuestionario de manejo del tiempo (García-Ros et al., 2008b), el cual es una adaptación al español del Time Management Behavior Questionarie-TMBQ (Macan et al., 1990) y está compuesto por 34 ítems con una escala de respuesta tipo likert del 1 al 5 , siendo adaptado en la presente investigación para la medición del tiempo personal de donde resultan 28 ítems.

Con el fin de conocer la confiabilidad del instrumento en la muestra estudiada se realizó un análisis factorial de componentes principales con rotación varimax y se estimó la confiabilidad a través del alfa de Cronbach. Posteriormente se realizaron los análisis estadísticos descriptivos y correlacionales a través del programa estadístico SSPS 18.

\section{Las competencias y la formación universitaria de profesionales de la administración}

Recientes investigaciones (Durán-García y Durán-Aponte, 2011; Ugarte y Naval 2010; Camperos, 2008; López, 2008; Capllonch y Castejón, 2007; Marzo et al., 2006; Cepeda, 1994) hacen referencia a la importancia de orientar la formación en todos los niveles educativos teniendo en cuenta un modelo basado en competencias, y coinciden en afirmar que es una posibilidad para lograr una formación integral del individuo, conjugando el saber ser, el saber hacer y el saber convivir durante el proceso de enseñanza y aprendizaje.

La formación por competencias permite abarcar el desarrollo de habilidades para la profesión, y además posibilita la transferencia de estas habilidades a los ámbitos en los que se desempeñe el individuo. Este enfoque permite la comprensión de este ser de manera integral, ya que considera cada elemento de su persona (habilidades, destrezas cognitivas, personalidad, talentos), sin desestimar alguno de ellos pues son importantes para afrontar cualquier reto, tanto personal como laboral.

Esta idea es respaldada por Tobón (2007) quien afirma que las competencias además de que buscan formar para responder ante las demandas social-empresariales, también tratan de contribuir a que las personas tengan una visión de futuro que contemple su desarrollo como ser humano, y después en lo laboral-empresarial para mejorar y transformar la realidad en la que están inmersos. 
Debido a la creciente producción de investigaciones relacionadas con el tema, también existen múltiples definiciones del término competencias y a su vez variadas clasificaciones, por lo tanto para los efectos del presente estudio se asume la definición de competencias aportada por Roegiers (2007) quien las considera como "un conjunto de capacidades ejercidas sobre determinados contenidos, en una categoría, clase o familia de situaciones para resolver los problemas que se presentan". Esta definición considera la utilidad de la competencia para dar soluciones a situaciones reales.

Se puede hablar entonces de un individuo que posee cierta y determinada competencia, siempre y cuando sea capaz de evidenciarla en cualquier situación de su vida que se le demande. Este carácter finalista y contextual de la competencia también lo declara Martínez (2011) como uno de los aspectos más relevantes de este enfoque de formación, pues además del desempeño en una tarea o actividad en un ámbito particular de trabajo, también se debe demostrar la integración de conocimientos, habilidades y actitudes en cualquier situación en donde se requiera.

Así mismo en el resumen de González y Wagenaar (2007:37) sobre las conclusiones del Proyecto Tunning para Latinoamérica, se expresa que existen dos tipos de competencias -aun cuando los términos empleados varíen de un autor a otro- "las relacionadas con un área de conocimiento (específicas de un campo de estudio), llamadas profesionales o técnicas y las competencias genéricas (comunes para diferentes cursos)". La capacidad para organizar y planificar el tiempo es considerada dentro del mencionado Proyecto como una competencia genérica de importante valor para distintas titulaciones de lberoamérica, entre ellas las relacionadas con la administración.

Escobar (2005), señala que las competencias genéricas (también llamadas por algunos autores directivas), contienen unas competencias de carácter propiamente empresariales a las que se denominan de eficacia personal e incluyen aquellos hábitos que facilitan una relación eficaz de la persona con su entorno. Entre ellas están la proactividad, el desarrollo personal y el autogobierno, este último integrado por la gestión personal del tiempo, del estrés, del riesgo, disciplina, concentración y autocontrol. Para Escobar (2005:40) "estas competencias miden la capacidad de autodirección, la cual resulta imprescindible para dirigir a otros...", elemento clave en la formación de profesionales de la administración.

Esta afirmación permite indicar que, un individuo que se desempeñará en un cargo administrativo, deberá ser capaz de autogestionar su propia conducta, regular sus acciones y gestionar su tiempo, de manera que pueda conducir, promover y dirigir los recursos personales, a favor de los cambios y el tiempo y los recursos de los otros que con el laboren.

Un adecuado manejo del tiempo no sólo es una competencia requerida por los empleadores para asegurar un buen uso de los recursos y el personal que se dispongan, sino que también es una forma de asegurar que el individuo podrá responder ante la presión y modificar las condiciones que le producen estrés para 
Competencia manejo del tiempo en la formación de profesionales de la administración Durán-Aponte, Emilse

preservar su salud física y mental, ya que tal como afirma Olvera (2008) se espera que los profesionales puedan enfocarse en las tareas laborales y usar su tiempo y energía de forma productiva a pesar de la presión. Estas afirmaciones dejan clara la importancia de tomar en cuenta la competencia manejo del tiempo en la formación profesional administrativa.

Ante esto no se pretende afirmar que el éxito y la efectividad de un buen profesional, gerente, directivo o coordinador depende sólo de las competencias, estas son importantes para describir estos aspectos pero, tal como lo afirmaba Boyatzis (1982) no suficientes para predecir el rendimiento de un profesional, es necesario tener en cuenta el contexto y las demandas de la actividad.

En vista de la relevancia de la formación en competencias genéricas y en específico de saber manejar o gestionar el tiempo como competencia de un buen profesional, resulta pertinente identificar aquellos elementos que la caracterizan y que son indicadores de presencia o ausencia de la misma en la actuación de un individuo.

\section{Manejo del tiempo}

En la actualidad son pocas las investigaciones Iberoamericanas que hacen referencia exclusiva al manejo del tiempo como una competencia requerida en profesionales que ocuparán cargos administrativos, a pesar de que tal como se mencionó en el apartado anterior, es una competencia necesaria dentro del conjunto de competencias genéricas que debe poseer un profesional.
Esto dificulta encontrar estudios recientes que enfaticen en las características de una persona competente en cuanto al manejo del tiempo, y mucho menos investigaciones que permitan establecer comparaciones producto de mediciones científicas.

Sin embargo algunas investigaciones hacen referencia del manejo del tiempo como una variable que incide en el estrés laboral (Wetthen y Cameron, 2005) y por consiguiente cuando no es bien manejada, desencadena conductas inadecuadas en el desempeño laboral, por lo cual es una prioridad que esta competencia se desarrolle dentro de las habilidades gerenciales. Otras investigaciones hacen referencia al manejo del tiempo como una variable que incide en el rendimiento académico (Durán-Aponte y Pujol, 2012; García-Ros y Pérez, 2009; García-Ros et al., 2008a; García-Ros et al., 2008b, García-Ros y Pérez, 2004; Barrera et al., 2008; Pérez et al., 2003), considerándola en muchos casos como un predictor eficaz del rendimiento y buen desempeño académico.

Por las características de las competencias se espera que quien las posea pueda transferirlas en cualquier situación que la demande, en el caso que nos ocupa, se espera que quienes puedan gestionar adecuadamente el tiempo personal para los estudios, también lleguen a hacerlo en su desempeño profesional.

Para efectos del presente estudio nos enfocaremos en los aspectos que permiten identificar el manejo del tiempo y las dimensiones que lo caracterizan. La competencia de manejo del tiempo es definida por García-Ros et al. (2008b), como un proceso en donde se establecen 
metas claras, se tiene en cuenta el tiempo disponible y se verifica el uso que se le da al tiempo. Esta definición comprende tres aspectos claves: 1) el establecimiento de metas, 2) las herramientas para la gerencia del tiempo y 3) la percepción de control o verificación del uso del tiempo personal.

Para Whetten y Cameron (2005), manejar eficazmente el tiempo significa que:

- Los individuos invierten su tiempo en asuntos importantes, no sólo en los urgentes.

- Son capaces de distinguir entro lo urgente y lo importante.

- Utilizan estrategias para gestionar el tiempo.

- Dicen "no" sin sentirse culpables.

Whetten y Cameron (2005), afirman que es necesario diferenciar entre las actividades importantes que son aquellas que producen un resultado deseado, cumplen con un fin valioso o logran un propósito significativo, y las actividades urgentes caracterizadas por la demanda de atención inmediata, se asocian con una necesidad expresada por alguien más o con un problema incómodo. Ante esto, una de las decisiones más difíciles para un manejo eficaz del tiempo es identificar y decidir entro lo importante y lo urgente.

Esto conlleva a alinear el manejo personal del tiempo con el logro de metas personales y por otro lado, con lograr más cosas a largo plazo que simplemente soluciones rápidas, lo cual lamentablemente no es una condición bien vista en el contexto Latinoamericano actual, sino las acciones efectivas que producen resulta- dos inmediatos aunque estos no sean duraderos o importantes, solo urgentes.

Así mismo Whetten y Cameron (2005), afirman que una forma de mejorar el manejo eficiente del tiempo es estar alerta ante las propias tendencias a utilizar el tiempo inapropiadamente. Por esta razón el estudio utiliza el Cuestionario de Manejo del Tiempo-TMBQ (Macan et al., 1990) adaptado por García-Ros et al., (2008b) para población de habla hispana, debido a que además de medir el establecimiento de objetivos y prioridades, las herramientas para la gestión del tiempo y la percepción de control del tiempo, también aporta información acerca de la preferencia por la desorganización que posee el individuo, lo cual es clave dentro del perfil de un profesional de la administración, ya que puede aportar tendencias inadecuadas y convertirse en un referente para realizar mejoras en la actuación.

Cada dimensión del instrumento está en sintonía con la definición de manejo del tiempo adoptada para este estudio la cual fue citada al inicio de este apartado, y las mismas se refieren a:

a. Establecer objetivos y prioridades: Evalúa la predisposición para selección, la capacidad para gestionar y priorizar las tareas que precisa hacer el individuo para alcanzar sus objetivos.

b. Herramientas para la gestión del tiempo: Mide el uso que el sujeto hace de las conductas asociadas usualmente con la gestión eficaz del tiempo, tales como técnicas donde se planifica un horario, listado para descartar tareas realizadas, uso de agenda. 
Competencia manejo del tiempo en la formación de profesionales de la administración Durán-Aponte, Emilse

c. Preferencias por la desorganización: Evalúa las formas en que el sujeto aborda sus tareas y el mantenimiento de un entorno de estudio planificado.

d. Percepción de control sobre el tiempo: Evalúa el grado en el que las percepciones del sujeto acerca del control del tiempo, afectan de forma directa al modo en cómo lo utiliza.

Es importante tener en cuenta que el manejo del tiempo posee un carácter contextual, por lo tanto dependerá de las características de la solicitud, asignación o tarea, el nivel de experticia que posea el individuo, el apoyo grupal, el manejo de herramientas que faciliten o agilicen el trabajo, delegar o compartir responsabilidades en caso de que sea necesario, y las consecuencias o repercusiones que tenga el cumplimiento o no de la asignación.

El profesional que desea manejar eficazmente su tiempo, debe conocer a donde quiere llegar, los recursos que posee para lograrlo, en cuanto tiempo puede hacerlo y como puede hacerlo mejor, esto implica tener una visión amplia de las debilidades y fortalezas reales que influyen en su planificación de futuro y lograr el establecimiento de metas claras.

Entonces, la formación en cuanto a herramientas para la planificación y el establecimiento de metas personales a corto, mediano y largo plazo, la configuración de un proyecto de vida, el autoconocimiento, así como el uso de herramientas para establecer horarios, agendar el tiempo de trabajo y el tiempo libre, son elementos que influirán positivamente en quienes se esfuercen por manejar eficazmente su tiempo, lo cual se verá evidenciado entre otras cosas, en el estableci- miento de objetivos y prioridades y la capacidad para diferenciar entre lo importante y lo urgente.

En función de lo establecido en cuanto a la formación universitaria por competencias, y las características de una buena gestión del tiempo en profesionales de la administración, resulta pertinente describir la manera en que estudiantes universitarios cercanos a egresar gestionan su tiempo, con el fin de establecer elementos o perfiles que permitan desarrollar futuras metodologías o estrategias que aborden el desarrollo de esta competencia.

\section{Manejo del tiempo y formación de profesionales de la administración}

Para obtener los resultados del presente estudio se tomaron en cuenta las variables género, carrera y los puntajes en las dimensiones del cuestionario. La Tabla 1 muestra la distribución de estudiantes por carrera y género, en donde la carrera de mayor frecuencia es Administración de Aduanas, lo cual es consistente con la población total de estudiantes y su distribución en carreras de la institución universitaria donde se realiza el estudio. En cuanto al género, son también las mujeres quienes presentan mayor frecuencia, lo cual no es un sesgo en la muestra pues es un aspecto común en las instituciones universitarias venezolanas asociadas a carreras administrativas.

Para conocer el perfil de los sujetos en el manejo del tiempo, se utiliza el Cuestionario de Manejo del Tiempo (García-Ros et al., 2008b). A partir de esto se puede observar en la Tabla 2 que la di- 
Tabla 1

Distribución de estudiantes por carrera y género

\begin{tabular}{lrrrrr}
\hline \multirow{2}{*}{ Carrera } & \multicolumn{2}{c}{ Sexo } & Total & \% Total \\
\cline { 2 - 3 } & Femenino & Masculino & & \\
\hline Admón. de Aduanas & 82 & 58 & 140 & 50,4 \\
Comercio Exterior & 47 & 21 & 68 & 24,5 \\
Admón. del Transporte & 8 & 8 & 16 & 5,8 \\
Org. Empresarial & 18 & 8 & 26 & 9,4 \\
Admón. del Turismo & 11 & 8 & 19 & 6,8 \\
Admón. Hotelera & 7 & 2 & 9 & 3,2 \\
Total & 173 & 105 & 278 & 100 \\
\hline
\end{tabular}

Fuente: Elaboración propia (2012).

Tabla 2

Matriz de correlación entre las dimensiones de manejo del tiempo

\begin{tabular}{lcccccc}
\hline \multicolumn{1}{c}{ Dimensiones } & $\mathrm{M}$ & $\mathrm{DT}$ & 2 & 3 & 4 & 5 \\
\hline 1. Objetivos/ Prioridades & 3,41 &, 623 &, $521^{* *}$ &,- 068 &,$- 146^{*}$ &,- 043 \\
2. Herramientas Gestión & 2,65 &, 998 & 1 &,- 062 &,- 038 &,- 078 \\
3. Preferencia desorganización & 2,21 &, 748 & & 1 &, $395^{* *}$ &, $129^{*}$ \\
4. Percepción de control & 2,66 &, 639 & & & 1 &, 038 \\
5.Información sobre manejo t. & - & - & & & & 1 \\
\hline
\end{tabular}

Fuente Elaboración propia (2012)

** La correlación es significativa al nivel 0,01 (bilateral). *La correlación es significante al nivel 0,05 (bilateral). M (media), DT (desviación típica), 2 (herramientas de gestión), 3 (preferencia desorganización), 4 (percepción de control), 5 (información sobre el manejo del tiempo).

mensión Establecimiento de objetivos y prioridades, posee un promedio $(\mathrm{M}=$ 3,41 ; DT=,623) mayor que el resto de las dimensiones. Esto representa un aspecto importante pues como ya se dijo, definir lo que se quiere lograr y reconocer las prioridades entre lo urgente y lo importante para el alcance de las metas personales, es un aspecto clave para el manejo eficaz del tiempo.

Le siguen las dimensiones Percepción de control y Herramientas de gestión con un promedio de $\mathrm{M}=2,66$ / DT=,639 y
$M=2,65 / D T=, 998$ respectivamente. Ambas dimensiones representan elementos clave para el manejo adecuado del tiempo, en vista de que el uso de herramientas (agendas, lista de tareas, horarios) demuestra un interés por gestionar el tiempo personal y a su vez, la percepción acerca de que este tiempo es controlado, puede contribuir a un incremento del uso de las herramientas.

En general, los participantes establecen objetivos y prioridades habitualmente, y algunas veces perciben que la 
Competencia manejo del tiempo en la formación de profesionales de la administración Durán-Aponte, Emilse

forma en la que manejan su tiempo afecta como lo utilizan. En cuanto a las herramientas disponibles para gestionar su tiempo, afirman utilizarlas algunas veces y pocas veces prefieren o tienden a la desorganización.

Estos resultados evidencian en general, la presencia de la competencia de manejo del tiempo en los estudiantes participantes de forma moderada, en vista de que las puntuaciones tienden hacia valores medios en la escala de puntuación establecida en el instrumento de medición (1-nunca al 5-siempre). Sin embargo es necesario detallar a profundidad, la forma en que se relacionan estas dimensiones entre ellas y con el resto de las variables.

Al realizar un análisis correlacional entre las dimensiones, solo se hallaron dos relaciones significativas. Se observa que existe una correlación positiva y moderada alta $(r=0,521 ; p=, 001)$ entre establecer objetivos y prioridades y el uso de herramientas de gestión del tiempo, lo que significa que en la medida que el futuro profesional perciba que puede y debe establecer prioridades, entonces hará más uso de las herramientas y técnicas disponibles para el manejo de su tiempo.

Al pensar en el manejo del tiempo como una competencia que requiere ser puesta en práctica día a día, la relación antes descrita conlleva a enfatizar en la importancia de motivar en los profesionales de la administración, el uso de herramientas que les permitan cumplir con las metas u objetivos trazados, sin desviarse por cuestiones urgentes o fáciles, sino atendiendo oportunamente lo que en un principio se tenga planificado.

De muy poco servirá establecer objetivos y prioridades si no se utilizan me- canismos para dejarlos por escrito, recordarlos, o simplemente descartarlos cuando ya estén cumplidos, en vista de que los elementos de la vida cotidiana que demandan esfuerzo y participación, podrían pasar desapercibidos sin que se note la inversión de tiempo realizada en ellos.

La dimensión preferencia por la desorganización presenta el menor promedio $(M=2,21$ / $D T=0,748)$, lo cual resulta satisfactorio pues evidencia que prefieren más la organización de sus tareas y actividades. Resulta interesante que entre esta dimensión y la dimensión de percepción de control existe una correlación positiva y moderada $(r=, 395 ; p=, 001)$, lo que permite indicar que en la medida en que existe una mayor tendencia hacia la desorganización, se percibe también una mayor necesidad por controlar el tiempo y remediar esta situación, lo que podría indicar que se está consciente de que actuar de forma desorganizada afecta el uso de su tiempo.

Este resultado es satisfactorio en vista de que se espera que el futuro profesional, tenga mínimas y si es posible ninguna preferencia por el desorden o la falta de control en cuanto a las tareas que realiza y el lugar donde lo hace. Además, que se espera que tenga conciencia de cómo actúa respecto a su tiempo y las consecuencias que esto puede traerle.

Adicionalmente, se consultaba en el cuadernillo si habían recibido información acerca del manejo del tiempo y solo 48 de ellos $(17,26 \%)$ afirmaron haber recibido información acerca del tema en la universidad (cursos vivenciales, propedéuticos, asesorías, cursaron el Ciclo de Iniciación Universitaria). Se debe tener claro que a pesar de que la mayoría indi- 
caba no haber recibido información o herramientas para gestionar su tiempo $(82,74 \%)$, también es cierto que los resultados promedio revelan un interés por establecer prioridades y priorizar sus en sus responsabilidades.

Así mismo en la Tabla 2 se evidencia una correlación positiva y baja $(r=, 129, p=, 005)$ entre los que no recuerdan haber recibido información del tema y los puntajes altos en la dimensión preferencia por la desorganización. Es importante este resultado ya que justifica la importancia de intensificar los programas de formación para promover un manejo adecuado del tiempo, indicado hacia donde se deben orientar los esfuerzos, ya que se espera erradicar aquellas conductas que evidencian una preferencia por la improvisación, salir de lo fácil, antes de lo importante o necesario y la desorganización en general.

Para determinar la relación entre las dimensiones de manejo del tiempo y las variables género y carrera, se aplicaron las pruebas de contraste de media (tStudent) y análisis de varianza (F-ANO$\mathrm{VA}$ ) respectivamente, presentadas en la Tabla 3. En lo que respecta a la relación entre el género y el manejo del tiempo, ambos grupos tienen puntajes aceptables de manejo del tiempo, sin embargo se observa que las mujeres presentan una media mayor que los hombres en las dimensiones establecimiento de objetivos $(M=3,45 / D T=, 58)$ y herramientas de gestión $(M=2,80 / D T=, 976)$ y esta relación se invierte en el caso de las dimensiones preferencia por la desorganización $(\mathrm{M}=2,27 / \mathrm{DT}=, 75)$ y percepción de control $(M=2,71 / D T=, 74)$ en las que los hombres presentan mayores puntajes promedio que las mujeres.

Esto puede significar una tendencia en los hombres por la desorganización de las tareas que realiza y el lugar donde las hace, teniendo en cuenta los resultados mencionados en el apartado anterior y las correlaciones entre las dimensiones. Sin embargo, solamente se observa una diferencia estadísticamente significativa entre mujeres y hombres en la dimensión herramientas de gestión (Diferencia de medias $=-, 195, p<001$ ), indicando que las mujeres evidencian un mayor uso de las herramientas de gestión. Estas herramientas pueden ser la agenda, los horarios, el listado de tareas, las notas recordatorias y otros.

Estos resultados no coinciden parcialmente con los aportes dados por Barrera et al. (2008), en su estudio de manejo del tiempo efectuado con estudiantes universitarios, en donde los hombres reportaban mejor desempeño en el manejo del tiempo que las mujeres.

En cuanto a las carreras y las dimensiones de manejo del tiempo no se encontraron diferencias significativas, sin embargo y a pesar de que no son estadísticamente significativas, se puede decir que los cursantes de la carrera Administración Hotelera mostraron el promedio más alto en establecimiento de objetivos $(\mathrm{M}=3,67 / \mathrm{DT}=, 69)$ y herramientas de gestión ( $M=3,04$ / $D T=, 811)$, mientras que los estudiantes de Comercio Exterior lo hicieron en percepción de control $(\mathrm{M}=2,77$ / $\mathrm{DT}=, 65$ ) y los de Administración del Turismo en preferencia por la desorganización $(M=2,31 / D T=, 79)$. 
Competencia manejo del tiempo en la formación de profesionales de la administración Durán-Aponte, Emilse

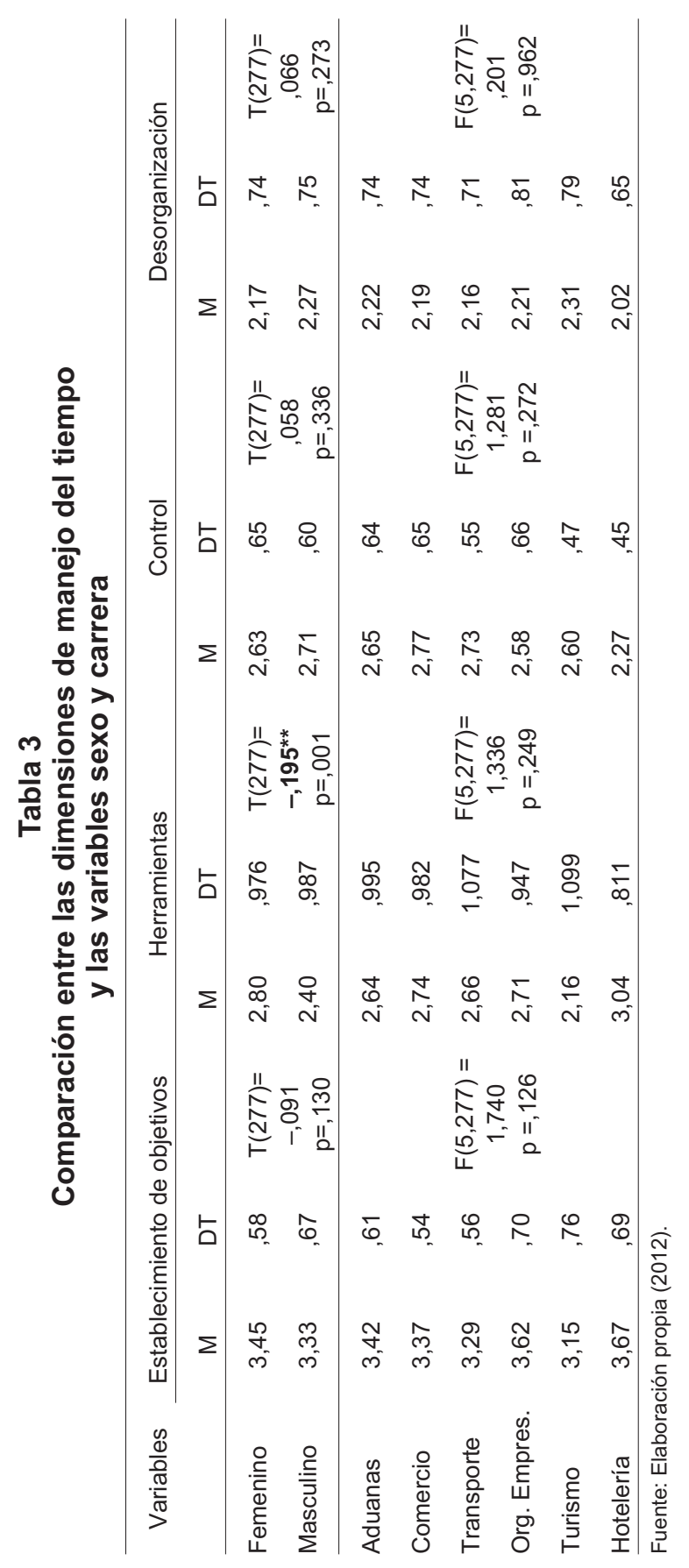




\section{Conclusiones}

Al considerar los resultados discutidos anteriormente, es necesario tener en cuenta que debido a las características del método empleado, procedimiento y muestra de la investigación, los datos aquí mostrados deben ser tomados como resultados iniciales y no como indicadores concluyentes; sin embargo, se puede afirmar que son consistentes desde el punto de vista teórico que sustenta el estudio.

Dado que el objetivo era describir las dimensiones de la competencia genérica manejo del tiempo en los estudiantes de administración, se puede decir que en los participantes en la investigación existe un perfil orientado al manejo del tiempo, pues se observa una mayor preferencia en la dimensión establecimiento de objetivos y prioridades, por lo tanto, podría esperarse que estos sujetos al insertarse en el ambiente de trabajo, sabrán manejar su tiempo adecuadamente y convertirse en un personal competitivo ante la demanda laboral.

Así mismo, las relaciones encontradas entre las dimensiones de manejo del tiempo dan cuenta de un proceso reflexivo y consciente en los estudiantes, con orientación hacia una búsqueda autorregulada del manejo de su tiempo, sin embargo, en cuanto a la diferencia de media significativa entre el género y las herramientas de manejo del tiempo, las mujeres tienen moderadamente mayores puntajes que los hombres. Este perfil orienta hacia donde y hacia quienes debe ir el énfasis en la formación de esta competencia.
Adicionalmente, una relación importante se encuentra entre la preferencia por la desorganización y los que no recuerdan haber recibido formación sobre el manejo del tiempo, ya que esto podría sugerir que de modo contrario, aquellos que si reciban formación podrían manejar su tiempo de forma eficaz, por lo tanto cobra importancia la idea de ejecutar propuestas de formación que desarrollen la competencia manejo del tiempo de modo transversal durante los estudios universitarios.

Tal como lo afirman Durán-Aponte y Durán-García (2012:74) "el énfasis está en integrar durante la formación de estos futuros profesionales, aquellas oportunidades y situaciones de aprendizaje que le permitan desarrollar rasgos de su personalidad, estilo de aprender y características personales, para ser utilizados posteriormente en el ámbito laboral".

El perfil obtenido en esta investigación a través de la descripción de las dimensiones de manejo del tiempo, permite diseñar propuestas que contribuyan con mejoras en esta competencia, fortaleciendo aquellas áreas débiles presentadas por los estudiantes, sobre todo en el caso de los hombres. Sin embargo, es importante tener en cuenta que son variadas las técnicas recomendadas para lograr un buen manejo del tiempo y estas no son del todo garantes de un desempeño eficaz, sino que es importante considerar el comportamiento de los demás y como esto puede también incidir en el desempeño individual.

Por otro lado, la descripción de este perfil en las dimensiones de manejo del tiempo dan cuenta de la ventaja del instrumento utilizado para obtenerlo, debido 
Competencia manejo del tiempo en la formación de profesionales de la administración Durán-Aponte, Emilse

a su sencillez de aplicación y las dimensiones que abarca, lo cual favorece su utilización en las actividades docentes y de investigación relacionadas con el tema, pero también puede contribuir a la gerencia del talento humano dentro de las organizaciones puesto que se convierte en un apoyo en el diagnóstico de las necesidades de formación de los empleados y en el diseño de programas de intervención.

De lo anterior se desprende la necesidad de sugerir para futuras investigaciones, profundizar en esta y otras competencias genéricas en profesionales en ejercicio, y así plantear modelos predictivos de un desempeño exitoso, estableciendo comparaciones entre aquellos factores que garantizan el éxito de todo profesional de la administración o gerente. También se recomienda considerar otras variables que puedan estar relacionadas con el manejo del tiempo, como la motivación de logro, la vocación, los estilos de personalidad, la responsabilidad y el dominio o no de la tarea a realizar.

\section{Referencias bibliográficas}

Boyatzis, R. (1982). The competent manager: a model for effective performance. New York: John Wiley \& Son.

Barrera, María, Donolo, Danilo y Rinaudo, María (2008). Ritmo de estudio y trayectoria universitaria. Anales de Psicología, Vol. 24, № 1, España, Servicio de Publicaciones de la Universidad de Murcia, pp. 9-15.

Camperos, M. (2008). La evaluación por competencias, mitos, peligros y desafíos. Educere, Revista Venezolana de Educación, Año 12, № 43, Venezuela, Universidad de los Andes, pp. 805-814.
Capllonch, Marta y Castejón, Francisco (2007). La adquisición de competencias genéricas a través de una comunidad virtual en el practicum. Teoría de la Educación y Cultura en la Sociedad de la Información Vol. 8, N ${ }^{\circ}$ 12, España, Universidad de Salamanca, pp. 168-187.

Castellano, Humberto (2010). Planificación: herramientas para enfrentar la complejidad, la incertidumbre y el conflicto. 2da. Ed. Venezuela, Cendes.

Cepeda, Jesús (1994). Metodología de la Enseñanza Basada en Competencias. Revista Iberoamericana de Educación. Año 4, № 34, España, Organización de Estados Iberoamericanos para la Educación, la Ciencia y la Cultura. Consultado el 06 de abril de 2010, disponible: http://www.rieoei. org/tec_edu28.htm

Durán-Aponte, Emilse y Durán-García, Martín (2012). Competencias sociales y las prácticas profesionales. Vivencias y demandas para la formación universitaria actual. Revista Cultura y Educación. Vol. 24, №1, España, Fundación Infancia y Aprendizaje, pp. 61-76.

Durán-Aponte, Emilse y Pujol, Lydia (2012). Estilos de aprendizaje, gestión del tiempo y rendimiento académico en estudiantes universitarios. V Congreso Mundial de Estilos de Aprendizaje, Santander, España, 27 y 28 de junio. (En prensa).

Durán-García, Martín y Durán-Aponte, Emilse (2011). Empresa, universidad y competencias. Propuesta de un modelo sistémico. Revista Gestión Universitaria. Vol. 3, $\mathrm{N}^{\circ} 3$, Argentina, Buenos Aires, Disponible en: http://www. gestuniv.com.ar/gu_03/v3n3a3.htm.

Escobar, Miriam (2005). Las competencias laborales: ¿La estrategia laboral para la competitividad de las organizaciones? Estudios Gerenciales, N ${ }^{\circ}$ 96, 
Colombia, Universidad ICESI, pp. 31-55.

González, J. y Wagenaar, R. (Eds). (2007). Tuning Educational Structures in Europe. Informe Final Fase Uno. Bilbao: Universidad de Deusto. Consultado el 09 de marzo de 2009, de: http://www. relint.deusto.es/TUNINGProject/spanish/doc_fase $1 /$ Tuning\%20Educational.pdf

García-Ros, Rafael y Pérez, Francisco (2009). Una aplicación web para la identificación de estudiantes de nuevo acceso en situación de riesgo académico (repositorios estratégicos y gestión del tiempo). Revista de Innovación Educativa, № 2, España, Universidad de Valencia, pp. 10-17.

García-Ros; Rafael, Pérez, Francisco; Talaya, Isabel (2008a). New university student's instruccional preferences and how these relate to learning stykes and motivational strategies. Electronic Journal of Research in Educational Psychology, Vol. 3, $\mathrm{N}^{\circ} 6$, España, Universidad de Almería, pp. 547-570.

García-Ros, Rafael, Pérez-González, Francisco, Talaya, Isabel.; \& Martínez, Estefanía (2008b). Analysis of Time Management Academic New students in the degree of Psychology: Predictive capacity and comparative analysis of two assessment instruments. International Journal of Developmental and Educational Psychology, Vol 1, № 2, España, Universidad de Barcelona, pp. 245-252.

García-Ros, Rafael \& Pérez-González, Francisco (2004). Evaluation of the Time Management Skill of Spanish High School Students: Factorial Structure and Relationship with Academic Achievement. School Psychology International, Vol. 2, $\mathrm{N}^{\circ} 25$, USA, pp. 167-184.
Hernández, Roberto, Fernández, Carlos y Baptista, Pilar (2003). Metodología de la investigación. (2a ed). México: McGrawHill.

Kerlinger, F. y Lee, H. (2008). Investigación del comportamiento: Métodos de investigación en ciencias sociales (4 ${ }^{\mathrm{a}}$ ed.). México.: McGraw-Hill.

López, Alejandro (2008). Origen y Fundamento de la Educación Basada en Competencias. Revista Xihmai, Vol. 3, México, Universidad La Salle, pp. 47-57.

Macan, Therese, Shahani, Comila, Dipboye, Robert, \& Phillips, Amanda (1990). College Students' Time Management: Correlations with Academic Performance and Stress. Journal of Educational Psychology, Vol. 82, N4, USA, University of Memphis, pp. 760768.

Martínez, J. (2011). El aprendizaje de competencias directivas en el marco del espacio europeo de educación superior, el caso del grado en turismo. Cuadernos de Educación y Desarrollo, Vol. 3 , N ${ }^{\circ} 5$, España, Universidad de Málaga, Consultado el 06 de febrero de 2011 de: http://www.eumed. net/rev/ ced/index.htm

Marzo, Mercedes, Pedraja, Marta, Rivera, Pilar (2006). Las competencias profesionales demandadas por las empresas: el caso de los Ingenieros. Revista de Educación N N 341 , España, Instituto Nacional de Evaluación Educativa, pp. 643-661.

Olvera, Gloria (2008). Las competencias más solicitadas en el mercado laboral de la bolsa universitaria de trabajo. Xihmai, Vol. 3, México, Universidad La Salle, pp. 13-23.

Pérez, Francisco; García, Rafael y Talaya, Isabel (2003). Estilos de aprendizaje y habilidades de gestión del tiempo académico en secundaria. Revista Portuguesa de Educacao. №16, 
Competencia manejo del tiempo en la formación de profesionales de la administración Durán-Aponte, Emilse

Portugal, Universidad do Minho, Vol. 1, pp. 59-74.

Rodríguez, Sebastián, Prades, Anna, Bernáldez, Lorena y Sánchez, Sergio (2010). Sobre la empleabilidad de los graduados universitarios en Catalunya: del diagnóstico a la acción. Revista de Educación, $N^{\circ} 351$, España, Instituto Nacional de Evaluación Educativa, pp. 107-137

Roegiers, X. (2007). Pedagogía de la integración. Competencias e integración de los conocimientos en la enseñanza. Costa Rica: Coordinación Educativa y Cultural Centroamericana y AECl.

Tejeda, Rafael (2011). Las competencias y su relación con el desempeño y la idoneidad profesional. Revista Iberoamericana de Educación, Año 10, №12, España, Organización de Estados Iberoamericanos para la Educación, la Ciencia y la Cultura, Disponible en: http://www.rieoei.org/deloslectores/ 3478Tejeda.pdf.
Tejeda, Rafael y Sánchez del Toro, Pedro (2009). Las competencias profesionales y su aprendizaje en la educación superior. Revista Pedagogía Universitaria Vol. XIV, No. 4, Cuba, Red de Centros de Estudios sobre Educación Superior, pp. 33-48

Tobón, Sergio (2007). El enfoque complejo de las competencias y el diseño curricular por ciclos propedéuticos. Acción Pedagógica, № 16, Venezuela, Universidad de los Andes, pp. 14-2 8.

Ugarte, Carolina y Naval, Concepción (2010). Desarrollo de competencias profesionales en la educación superior. Un caso docente concreto. Revista Electrónica de Investigación Educativa (Número Especial). Consultado el 04 de agosto de 2010, en: http://redie. uabc.mx/contenido/NumEsp2/contenido-ugarte.html

Whetten, David y Cameron, Kim (2005). Desarrollo de Habilidades Directivas. 6ta Edición. México: Pearson. 